\title{
Medicinal herbs and bioactive compounds overcome the drug resistance to epidermal growth factor receptor inhibitors in non-small cell lung cancer (Review)
}

\author{
HIU YAN JENNIFER LEE ${ }^{1}$, MINGJING MENG ${ }^{2}$, YULONG LIU ${ }^{1}$, \\ $\mathrm{TAO}^{2} \mathrm{SU}^{2}$ and HIU YEE KWAN ${ }^{1}$ \\ ${ }^{1}$ School of Chinese Medicine, Hong Kong Baptist University, Hong Kong, SAR; \\ ${ }^{2}$ International Institute for Translational Chinese Medicine, School of Pharmaceutical Sciences, \\ Guangzhou University of Chinese Medicine, Guangzhou, Guangdong 510006, P.R. China
}

Received April 17, 2021; Accepted June 4, 2021

DOI: 10.3892/ol.2021.12907

\begin{abstract}
Lung cancer is the leading cause of cancer-related mortality worldwide. Non-small cell lung cancer (NSCLC) accounts for $\sim 85 \%$ of all lung cancer cases. Patients harboring epidermal growth factor receptor (EGFR) mutations usually develop resistance to treatment with frontline EGFR-tyrosine kinase inhibitors (EGFR-TKIs). The present review summarizes the current findings and delineates the molecular mechanism of action for the therapeutic effects of herbal extracts and phytochemicals in overcoming EGFR-TKI resistance in NSCLC. Novel molecular targets underlying EGFR-TKI resistance in NSCLC are also discussed. This
\end{abstract}

Correspondence to: $\mathrm{Dr}$ Tao $\mathrm{Su}$, International Institute for Translational Chinese Medicine, School of Pharmaceutical Sciences, Guangzhou University of Chinese Medicine, 232 Waihuan East Road, Guangzhou, Guangdong 510006, P.R. China

E-mail: sutao@gzucm.edu.cn

Dr Hiu Yee Kwan, School of Chinese Medicine, Hong Kong Baptist University, 7 Baptist University Road, Kowloon Tong, Hong Kong, SAR, P.R. China

E-mail: hykwan@hkbu.edu.hk

Abbreviations: CIP2A, cancerous inhibitor of protein phosphatase 2A; EGFR, epidermal growth factor receptor; ERK, extracellular signal-regulated kinase; MMP, matrix metalloproteinase; MTA-1, metastasis-associated protein-1; mTOR, mammalian target of rapamycin; NF- $\kappa \mathrm{B}$, nuclear factor- $\kappa \mathrm{B}$; NSCLC, non-small cell lung cancer; PI3K, phosphatidylinositol 3-kinase; PFS, progression-free survival; PP2A, protein phosphatase-2A; ROS, reactive oxygen species; SHP2, SH2 domain-containing phosphatase-2; STAT3, signal transduction and activator of transcription 3; TKI, tyrosine kinase inhibitor; USP8, ubiquitin-specific peptidase 8; VEGF, vascular endothelial growth factor; COX-2, cyclooxygenase-2; $\mathrm{CDK}$, cyclin-dependent kinase

Key words: non-small cell lung cancer, epidermal growth factor receptor, herbal medicine, Chinese medicine, drug resistance review provides valuable information for the development of herbal bioactive compounds as alternative treatments for EGFR-TKI-resistant NSCLC.

\section{Contents}

1. Introduction

2. Clinical challenges of treating NSCLC

3. Treatment with medicinal herbs prolongs the PFS time for patients with advanced NSCLC treated with EGFR-TKIs

4. Herbal bioactive compounds can overcome drug resistance to EGFR-TKIs and enhance anti-EGFR therapeutic effects in NSCLC

5. Perspective

6. Conclusion

\section{Introduction}

Lung cancer is the most commonly occurring cancer worldwide, and $\sim 6.3 \%$ of the population will be diagnosed with lung or bronchial cancer at some point during their lifetime (1).

Lung cancer can be categorized into small cell lung cancer (SCLC) and non-SCLC (NSCLC). NSCLC is a type of epithelial cancer that accounts for $\sim 85 \%$ of all lung cancer cases (2). As reported by the American Cancer Society, based on the individuals diagnosed with NSCLC between 2010 and 2016, the 5-year survival rate for NSCLC is $63 \%$ for patients with localized tumors, but only $35 \%$ for those with early-stage tumors and $7 \%$ for those with distant tumors (2). Among the different types of NSCLC, the most common types are squamous cell carcinoma, large cell carcinoma and adenocarcinoma (2). Squamous cell carcinoma refers to cancer arising in the flat cells lining the inside of the airways of the lungs (2). Large cell carcinoma develops in the outer regions of the lungs and the cancer cells are large in size when viewed under the microscope (2). Lung adenocarcinoma is a subtype that begins in the cells lining the alveoli (2). 


\section{Clinical challenges of treating NSCLC}

NSCLC is the most common subtype of lung cancer. In total, $10-35 \%$ of NSCLC cases harbor activating epidermal growth factor receptor (EGFR)-mutations (3). EGFR mutations in NSCLC have given rise to the opportunity to use EGFR-tyrosine kinase inhibitors (TKIs) for treatment, and these inhibitors have become one of the frontline treatments for NSCLC. First-generation EGFR-TKIs include gefitinib and erlotinib, which reversibly bind to the ATP-binding site of the intracellular TK-domain of EGFR and impede EGFR autophosphorylation and the subsequent activation of the downstream signaling pathways. Second-generation EGFR-TKIs include afatinib and dacomitinib, which not only inhibit the TK-domain of EGFR, but also inhibit other members of the EGFR family such as the ErbB receptors (4).

Several clinical studies have, however, reported adverse events involving EGFR-TKI treatments. For example, gefitinib induces diarrhea $(5,6)$, causes various levels of hepatotoxicity (7), and results in liver dysfunction (7), skin disorders (8), stomatitis and mucositis (8). Furthermore, EGFR-TKI-induced paresthesia causes insensitivity and numbness of the limbs $(7,8)$. In more severe cases, the patients will lose sensation $(7,8)$. The third-generation EGRF-TKI, osimertinib, also causes grade 3 adverse events, as reported in a previous clinical trial (ClinicalTrials.gov number, NCT02296125) (9).

In addition to the reported side effects, the initial response to EGFR-TKIs is transient and most patients develop resistance to EGFR-TKI treatment, with a median progression-free survival (PFS) time of only 9-13 months $(10,11)$. With regard to the intrinsic resistance to EGFR-TKIs, genomic profiling of tumor specimens by high-throughput next generation sequencing analyses has highlighted gene mutations, including single nucleotide variants, point mutations, gene insertions and deletions, copy number variations and dysregulated expression of oncogenes (4,12-14). Furthermore, activation of alternative signaling pathways and phenotypic transformation of NSCLC to SCLC has also led to the development of drug resistance (11).

More importantly, $40 \%$ of lung cancer patients are diagnosed at the advanced stage (2), making treatment difficult. In a study analyzing data from patients enrolled in the Surveillance, Epidemiology and End Results (SEER) cancer registry, patients with stage III NSCLC treated with chemo-radiotherapy had a 3-year survival probability of only $24.1 \%$ (15). Although clinical results show that the first-line treatment with EGFR-TKIs significantly enhances the PFS time compared with standard chemotherapy in patients with advanced NSCLC harboring EGFR mutations, the reported adverse events and the development of drug resistance remain a clinical challenge for treatment.

\section{Treatment with medicinal herbs prolongs the PFS time for patients with advanced NSCLC treated with EGFR-TKIs}

With advancements in technology, recent studies have shown that treatment with medicinal herbs is an effective adjuvant to anti-EGFR therapy for the treatment of advanced NSCLC $(16,17)$. Compared with EGFR-TKI monotherapy, the combination of herbal medicine and EGFR-TKIs achieves a significantly higher objective response rate [risk ratios (RR), 1.34; 95\% CI, 1.15-1.57; $\mathrm{P}=0.0002$ ], disease control rate (RR, 1.8; 95\% CI, 1.09-1.27; $\mathrm{P}<0.0001)$, 1-year survival rate (RR, 1.21; 95\% CI, 1.01-1.44; $\mathrm{P}=0.04)$ and 2-year survival rate (RR, 1.91; 95\% CI, 1.26-2.89; $\mathrm{P}=0.002$ ), and improves the Karnofsky performance status (RR, 1.38; 95\% CI, 1.26-1.51; $\mathrm{P}<0.00001)(18)$. Another clinical trial reported that the median PFS time was significantly longer in the herbal medicine and EGFR-TKI combination treatment group (13.50 months; 95\% CI, 11.20-16.46) compared with that in the EGFR-TKI mono-treatment group (10.94 months; 95\% CI, 8.97-12.45) (hazard ratio, 0.68; 95\% CI, 0.51-0.90; $\mathrm{P}=0.0064)$ (17). Furthermore, population-based cohort studies clearly demonstrate that adjunctive herbal medicine improves the survival of patients with advanced-NSCLC who have received EGFR-TKI treatment (19-23).

Brucea javanica is a well-known medicinal herb in Asia. Clinical research shows that $B$. javanica not only reduces the incidence of nausea, vomiting and leukopenia in patients with advanced NSCLC (24), but that it also potentiates the efficacy of EGRF-TKI treatment in NSCLC harboring L858R/T790M EGFR mutations (25). It is also a safe adjuvant option to chemotherapy that can maximize the chemotherapeutic effects for NSCLC treatment (26). Marsdenia tenacissima (Roxb.) Wight et Arn., is also commonly used in herbal medicine. Experimental research shows that $M$. tenacissima improves the sensitivity of TKI-resistant NSCLC cells to gefitinib treatment. The combination of $M$. tenacissima and gefitinib significantly potentiates the inhibitory effect of gefitinib on cancer growth by inhibiting the signaling pathways that are activated by EGFR, such as the phosphatidylinositol 3-kinase (PI3K)/Akt, mammalian target of rapamycin (mTOR) and Ras/Raf/mitogen-activated protein kinase/extracellular signal-regulated kinase (ERK) kinase/ERK signaling cascades $(27,28)$. Another study suggested that $M$. tenacissima treatment overcomes EGFR-TKI resistance by inhibiting the receptor tyrosine kinases c-MET and Axl. MET and Axl are activated by hepatocyte growth factor and growth arrest-specific 6, respectively. Upon activation, c-MET and Axl regulate the key processes in tumorigenesis implicated in EGFR activation (29). Combined M. tenacissima and EGFR-TKI treatment significantly inhibits the c-Met and Axl-activated signaling pathways and overcomes erlotinib and gefitinib resistance in NSCLC (30). With regard to the C-21 steroidal glycosides isolated from $M$. tenacissima, some have anticancer effects $(31,32)$. However, the bioactive compounds in M. tenacissima that reverse EGFR-TKI resistance in NSCLC have not been identified. Herba epimedium (Horny Goat Weed) is a Chinese medicinal herb. A previous study showed that the combined treatment of Epimedium koreanum Nakai extract and gefitinib had an antiproliferative effect in T970M EGFR-resistant NSCLC cells (33). Further in vitro and in vivo studies showed that the extract decreased the phosphorylation of EGFR family members such as EGFR, HER-2, HER-3 and EGFR, and inhibited the downstream signaling pathway PI3K/Akt/mTOR, thus inhibiting the EGFR-resistant cell migration, invasion, angiogenesis and other transformation activities $(34,35)$. 


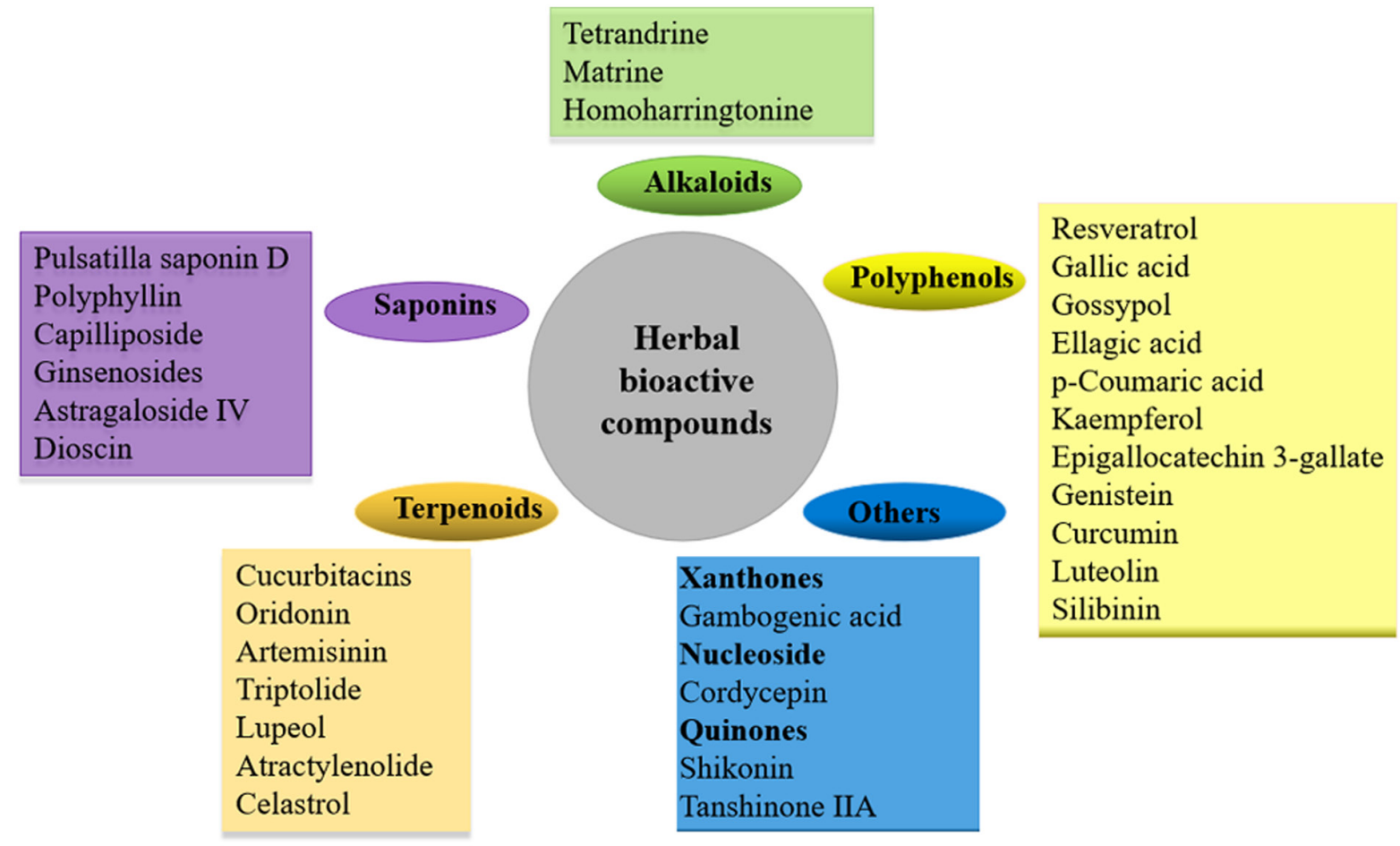

Figure 1. Herbal bioactive compounds that potentiate the therapeutic effects of EGFR-TKIs. Different natural products are marked in different colors. The green box presents the alkaloids, the purple box presents the saponins, the orange box presents the terpenoids, the yellow box presents the polyphenols and the blue box presents the other compounds, including the resins, nucleosides and quinones.

4. Herbal bioactive compounds can overcome drug resistance to EGFR-TKIs and enhance anti-EGFR therapeutic effects in NSCLC

A number of plant-derived compounds serve as the basis for the development of evidence-based pharmaceutical drugs. Studies have demonstrated that some of the bioactive compounds in medicinal herbs overcome drug resistance to EGFR-TKIs and potentiate the therapeutic effects of EGFR-TKIs. These compounds include polyphenols, saponins, terpenoids, alkaloids, quinones, resins and nucleosides (Fig. 1).

\section{Polyphenols}

Resveratrol. Resveratrol belongs to a class of polyphenolic compounds called stilbenes. This natural polyphenol can be detected in $>70$ plant species (36). Resveratrol decreases CYP1A1 and ABCG2 expression, and impairs gefitinib elimination from cells, increasing intracellular gefitinib concentration, which helps to overcome gefitinib resistance (37). The accumulated gefitinib triggers apoptosis, autophagy and senescence in gefitinib-resistant NSCLC cells (37). Therefore, co-treatment with gefitinib and resveratrol potentiates the anti-NSCLC effects of gefitinib.

Gallic acid. Gallic acid is a member of the hydroxybenzoic acids commonly found in a number of herbs and fruits. Gallic acid accelerates EGFR turnover (38), inhibits the Src-signal transducer and activator of transcription-3 (STAT3) signaling pathway, and induces apoptosis and cell cycle arrest in EGFR-TKI-resistant NSCLC, but not in EGFR-TKI-sensitive NSCLC, suggesting that gallic acid overcomes the acquired resistance to EGRF-TKI resistance in NSCLC (39).

Curcumin. Curcumin, also called diferuloylmethane, is isolated from the rhizome of Curcuma longa. Curcumin has anti-NSCLC effects; it inhibits NSCLC cell proliferation and induces apoptosis by increasing caspase-3 activity and the expression of miR-192-5p, which suppresses the phosphoinositide 3-kinase (PI3K)/Akt signaling pathway in NSCLC (40). miR-192-5p mimics enhance the effects of curcumin on cell viability and apoptosis, and suppress the PI3K/Akt signaling pathway in NSCLC cells, while anti-miR-192-5p mimics reverse the effect of curcumin on NSCLC cells (40). Curcumin also suppresses the proliferation and invasion of NSCLC by inhibiting the metastasis-associated protein-1 (MTA-1)-activated Wnt/ $\beta$-catenin pathway (41). MTA-1 promotes NSCLC cell invasion and metastasis (42). The decrease in MTA-1 by curcumin treatment inhibits NSCLC growth. Furthermore, curcumin induces autophagy in NSCLC and the autophagy inhibitor 3-methyladenine (3-MA) partly blocks the inhibitory growth effect of curcumin on cancer cells (43).

Curcumin also potentiates the therapeutic effects of gefitinib. Curcumin induces apoptosis in TKI-resistant NSCLC cells by decreasing EGFR phosphorylation and increasing EGFR degradation, thus inhibiting cancer growth (44). More importantly, the combination treatment of curcumin and EGFR-TKI markedly inhibits NSCLC growth by decreasing the expression of EGFR, c-MET and cyclin D1. The combination treatment confers a better survival rate and decreases intestinal mucosal damage in intestinal epithelial cells by regulating mitogen-activated protein kinase activity (45). 
Furthermore, the combination of curcumin and gefitinib causes marked autophagy induction, autophagic cell death and autophagy-mediated apoptosis when compared with curcumin or gefitinib treatment alone. Pharmacological autophagy inhibitors bafilomycin A1 or 3-MA, or knockdown of beclin-1 or autophagy-related 7, ameliorate treatment-induced autophagic cell death (45). Erlotinib and afatinib are other EGFR-TKIs used for frontline treatment. Co-administration of erlotinib and curcumin significantly decreases NSCLC cell viability by inducing apoptosis and increasing the expression of IкB (46), which restricts nuclear factor $-\kappa \mathrm{B}(\mathrm{NF}-\kappa \mathrm{B})$ to the cytoplasm and inhibit its DNA binding activity (47). The combination treatment also significantly increases apoptosis by decreasing the expression of EGFR and survivin, and inhibiting NF- $\kappa \mathrm{B}$ activity in erlotinib-resistant NSCLC cells (48). The application of curcumin and afatinib as a combination treatment for gefitinib- and erlotinib-resistant NSCLC has already been patented (CN105476996A).

Curcumin also overcomes chemotherapeutic resistance in NSCLC. A recent study implicated HIF-1 $\alpha$ in the development of chemotherapeutic resistance in cancer; therefore, targeting HIF-1 $\alpha$ either by RNA-interference or small interfering RNA may overcome the resistance to cisplatin (49). It has been reported that combined curcumin and cisplatin treatment markedly inhibits cisplatin-resistant NSCLC cell proliferation and triggers apoptotic death, by promoting HIF-1 $\alpha$ degradation and activating caspase-3, respectively (49). Curcumin also reverses cisplatin resistance by increasing cisplatin-induced apoptosis by inducing generation of intracellular reactive oxygen species (ROS) and proteosomal degradation of Bcl-2 in NSCLC (50).

\section{Saponins}

Ginsenosides. Ginsenosides are the main saponins in ginseng roots, fruits, stems and leaves. To date, $>60$ ginsenosides have been isolated and identified. Although the basic structure of ginsenosides are similar, the different arrangements in the four rings of steroid nuclei are different in different sub-types, such as the A-Panaxadiol group (e.g., Rb1, Rb2, Rb3, Rc, Rd, Rg3 and Rh2), the B-Panaxatriol group (e.g. Re, Rg1, Rg2 and Rh1) and the C-Oleanolic acid group (e.g. Ro) (51). One study showed that ginsenoside upregulated vaccinia-related kinase 1 and activated the tumor suppressor $\mathrm{p} 53$-binding protein-1, thus inhibiting NSCLC cell proliferation (52).

Ginsenosides also promote the antitumor activity of gefitinib in NSCLC. It has been demonstrated that ginsenoside Rg3 increases the cytotoxic effects of gefitinib in NSCLC cells in a dose- and time-dependent manner (53). The combination treatment of gefitinib and ginsenoside $\operatorname{Rg} 3$ significantly increases apoptosis in NSCLC cells by increasing the expression of the pro-apoptotic protein Bax and the activity of caspase-3, and by decreasing the expression of the anti-apoptotic protein Bcl-2 (53). The combination treatment also inhibits NSCLC cell migration by decreasing the protein expression of Snail and Slug (53), which are master regulators that regulate numerous genes that contribute to epithelial-mesenchymal transition (EMT), including E-cadherin (54). Osimertinib is the third-generation EGFR-TKI. A recent study revealed that osimertinib-resistant NSCLC cells displayed stronger stemness than the parental cells, which conferred to them greater metastatic potential (55). Notably, ginsenoside $\operatorname{Rg} 3$ attenuates the stemness of NSCLC cells and the resistance to osimertinib, as evidenced by the decreased expression of stemness markers and decreased spheroid formation ability, which is dependent on the Hippo signaling pathway (56). Furthermore, ginsenosides overcome resistance to chemotherapy drugs such as cisplatin (DDP), which is used for the treatment of NSCLC. Clinical research shows that ginsenoside Rg3 enhances the therapeutic effects of chemotherapy in patients with NSCLC. $\mathrm{Rg} 3$ treatment prolongs the overall survival time and improves the quality of life for the patients (57). $\mathrm{Rg} 3$ restores leucocyte count, decreases vascular endothelial growth factor (VEGF) expression and increases the CD4/CD8 T-cell ratio in these patients. Clinical outcomes show that $\operatorname{Rg} 3$ combined with chemotherapy enhances the efficacy and overall survival time of patients with NSCLC. Experimental research also suggests that ginsenoside overcomes the resistance to cisplatin in NSCLC. In cisplatin-resistant NSCLC (A549/DDP) cells, ginsenoside inhibits the NRF2 pathway and significantly sensitizes A549/DDP cells to cisplatin. NRF2 knockdown attenuates the synergistic effects of ginsenoside in cisplatin-resistant cells (58). A previous study also reported that $\mathrm{Rg} 3$ alleviated the resistance of A549/DDP cells to cisplatin treatment by decreasing PD-L1 expression via the inhibition of NF- $\mathrm{NB}$ p65 and Akt; therefore, restoring the cytotoxicity of $\mathrm{T}$ cells to the cancer cells (59).

Astragaloside IV. Astragaloside IV is a bioactive saponin found in the roots of a Chinese medicinal herb Astragalus membranaceus (Fisch.) Bge. Astragaloside IV sensitizes NSCLC to gefitinib by regulating sirtuin 6 (SIRT6), and inhibiting SIRT6 abolishes the sensitization role of astragaloside IV in NSCLC cells including HCI-H1299, HCC827 and A549 cells (60). SIRT6 is an NAD-dependent deacetylase and promotes the metastasis of NSCLC. Depletion of SIRT6 inhibits transforming growth factor- $\beta 1$-induced EMT in NSCLC cells, which underscores how astragaloside IV potentiates the anti-NSCLC effects of gefitinib.

Polyphyllin (PPI). PPI, PPII and PPVII are the main active saponins isolated from Paris polyphylla. PPI overcomes gefitinib resistance in NSCLC cells by decreasing cell viability and inducing apoptosis via downregulation of MALAT long non-coding RNA and inactivating the STAT3 signaling pathway (61). Both MALAT and STAT3 promote NSCLC growth. Overexpression of MALAT has been associated with a poor prognosis in patients with NSCLC (62), including recurrence and metastasis $(63,64)$. Mechanistic studies show that MALAT activates Akt/mTOR signaling in NSCLC and promotes cancer cell migration and invasion. High expression of STAT3 in patients with NSCLC is also associated with a poor prognosis $(65,66)$, and mediates drug resistance to EGFR-TKIs (67). Therefore, downregulating these two signaling molecules potentiates the anti-NSCLC effects of gefitinib. PPII restores sensitization of resistant NSCLC cells to gefitinib by inhibiting the PI3K/Akt/mTOR signaling pathway, thus potentiating the anti-proliferative effects of gefitinib and gefitinib-induced apoptosis (68). PPVII enhances the anti-proliferative effects of gefitinib and gefitinib-induced $\mathrm{G}_{1}$ phase arrest by modulating the $\mathrm{p} 21$ signaling pathway 
in gefitinib-resistant NSCLC cells (69). Another study by Lou et al also reported that PPI overcame erlotinib resistance in NSCLC by reversing EMT and modulating the IL-6/STAT3 signaling pathway. Co-treatment of erlotinib and PPI completely abrogated the growth of NSCLC (70).

Both PPI and PPVII have a chemo-sensitizing effect; they overcome cisplatin resistance in NSCLC by enhancing apoptosis, reversing EMT, suppressing the cancerous inhibitor of protein phosphatase $2 \mathrm{~A}(\mathrm{CIP} 2 \mathrm{~A}) / \mathrm{AKT} / \mathrm{mTOR}$ signaling axis and activating autophagy in cancer cells $(71,72)$.

Dioscin. Dioscin is a natural steroidal saponin abundantly found in Dioscorea nipponica Makino. Dioscin overcomes EGFR-TKI resistance in NSCLC by downregulating the expression of SH2 domain-containing phosphatase-2 (SHP2) at the transcriptional level through the induction of ROS production, and therefore decreases the binding of p53 to the SHP2 promoter (73). It is known that SHP2 is required for the growth of KRAS-mutant NSCLC, and the inhibition of SHP2 in KRAS-mutant NSCLC provokes a senescence response (74).

Capilliposide. Capilliposide is a saponin in the Chinese medicinal herb Lysimachia capillipes; it inhibits AKT activity, increases the pro-apoptotic effect of gefitinib and decreases EGFR phosphorylation in gefitinib-resistant NSCLC cells (75).

\section{Terpenoids}

Cucurbitacins. Cucurbitacins, such as cucurbitacin B and D, are tetracyclic triterpenes isolated from the Cucurbitaceae plant family. Cucurbitacin B potently suppresses NSCLC growth by inducing $\mathrm{G}_{2} / \mathrm{M}$ cell-cycle arrest and mitochondrial apoptosis. The anti-NSCLC effects of cucurbitacin B are attenuated by the thiol antioxidant $\mathrm{N}$-acetylcysteine, while glutathione synthesis inhibitor butithione-sulfoxime exacerbates the anti-NSCLC effects (76). Further research suggests that NSCLC cells treated with cucurbitacin B have a significant decrease in the ratio of thiols and glutathione to oxidized glutathione, suggesting that disruption of the cellular redox balance mediates the anti-NSCLC effects of cucurbitacin B (77).

Cucurbitacin B and D also overcome gefitinib resistance in NSCLC cells. CIP2A inhibits protein phosphatase-2A (PP2A) (78), which suppresses tumor growth through dephosphorylation of oncogenic kinases and transcription factors (79). Cucurbitacin B suppresses the CIP2A/PP2A/Akt signaling axis in gefitinib-resistant cells and inhibits cancer growth and metastasis (80). Furthermore, the cucurbitacin B-mediated inhibition of the CIP2A/PP2A/Akt axis also induces the lysosomal degradation of EGFR in NSCLC cells (80). In a previous study, cucurbitacin D overcame gefitinib resistance by blocking EGF binding to EGFR, as demonstrated by a solid-phase EGF-EGFR interaction assay. In the analysis, cucurbitacin D directly inhibited the interaction between EGF and EGFR (81). Hence, cucurbitacin D decreases EGFR phosphorylation in gefitinib-resistant NSCLC cells (81).

Oridonin. Oridonin is a diterpenoid compound extracted from Isodon plant Rabdosia rubescens, Isodon japonicus
Hara and I. trichocarpus (82). Oridonin inhibits the growth of gefitinib-resistant NSCLC cells. Oridonin decreases the expression of matrix metalloproteinase-12 (MMP-12) and CIP2A, suggesting that oridonin inhibits the growth and metastasis of gefitinib-resistant NSCLC by suppressing the EGFR/ERK/MMP-12 and CIP2A/Akt signaling pathways (83).

Artemisinin. Artemisinin is a sesquiterpene lactone in the Chinese medicinal herb Artemisia annua L. In EGFR-TKI-resistant NSCLC cells, artemisinin induces apoptosis and cell cycle arrest at the $G_{1}$ phase. Artemisinin decreases $\mathrm{Wnt} / \beta$-catenin protein levels by stimulating the release of naked cuticle homolog 2 (NKD2) and Axin2 (84). Both NKD2 and Axin2 are negative regulators of the Wnt/ $\beta$-catenin signaling pathway. Downregulation of NKD2 causes Wnt activation and increases the invasive potential of NSCLC (85). Axin promotes the phosphorylation and the consequent degradation of $\beta$-catenin, and thus suppresses the Wnt/ $\beta$-catenin signaling pathway (86). One study reported that the combination of artemisinin and onconase, a RNase of ribonuclease A, synergistically inhibited NSCLC growth (87). However, whether the combination of artemisinin and onconase can overcome gefitinib resistance in NSCLC is not known.

Dihydroartemisinin is the semi-synthetic derivative of artemisinin. It has been reported that treatment with dihydroartemisinin and gefitinib synergistically inhibits NSCLC cell growth and induces apoptosis via the Akt/mTOR/STAT3 pathway. Furthermore, this combination of treatment induces NSCLC cell cycle arrest in the $\mathrm{G}_{2} / \mathrm{M}$ phase, which is associated with decreased expression of $\mathrm{G}_{2} / \mathrm{M}$ regulatory proteins, including cyclin B1 and cyclin-dependent kinase 1. Combined treatment with dihydroartemisinin and gefitinib also significantly decreases the metastatic potential of NSCLC compared with treatment with gefitinib alone (88).

\section{Alkaloids}

Matrine. Matrine is an alkaloid isolated from Sophora flavescens. Treatment with a combination of matrine and afatinib, an EGFR-TKI, results in increased growth inhibitory effects of NSCLC cells by inhibiting the JAK/STAT3 signaling pathway (89), suggesting that matrine can overcome drug resistance to afatinib in NSCLC.

Furthermore, matrine also overcomes resistance to chemotherapy. In cisplatin-resistant NSCLC cells, matrine inhibits $\beta$-catenin activation and survivin expression, and induces apoptotic death, coupled with the loss of mitochondrial membrane potential and activation of caspase-9 and -3 (90).

Oxymatrine. Oxymatrine is one of the alkaloid components in Sophora flavescens; it inhibits NSCLC growth by suppressing the EGFR signaling pathway by inhibiting the activity of wild-type EGFR and EGFR with exon 19 deletion and L858R/T790M mutation (91). Further studies are needed to verify whether oxymatrine overcomes EGFR-TKI resistance in NSCLC.

\section{Quinonoids}

Shikonin. Shikonin is a naphthoquinone compound extracted from the root of the Chinese medicinal herb 
Lithospermum erythrorhizon. Shikonin exhibits cytotoxicity, increases ROS production and induces apoptosis in NSCLC cells (92). Inhibiting ROS production blocks shikonin-induced apoptosis (92). Furthermore, shikonin also induces necroptosis, which is necrosis in a programmed fashion, mediated by signal transduction from receptor-interacting serine/threonine kinase (RIP)-1 to RIP-3. It has been reported that shikonin increases the expression of RIP-1 and induces necroptosis in NSCLC (93). Notably, the shikonin-induced necroptosis is enhanced by the inhibition of autophagy in NSCLC cell (93). Shikonin also sensitizes NSCLC to EGFR-TKI treatment. Shikonin in combination with erlotinib or gefitinib significantly enhances ROS-mediated apoptosis in NSCLC when compared with EGFR-TKI treatment alone $(92,94)$. Furthermore, the combination of shikonin and gefitinib has synergistic anticancer effects via the inhibition of pyruvate kinase M2 (PKM2) and its downstream signaling molecules, including STAT3 and cyclin D1, in NSCLC cells (95). However, there is lack of consistent evidence suggesting the correlation of PKM2 and tumor grade in NSCLC (96). Another study has suggested that shikonin sensitizes gefitinib-resistant NSCLC cells by enhancing TRAIL-induced cytotoxicity via modulation of the STAT3, c-Jun N-terminal kinase and AKT signaling pathways (97). Preclinical research has shown that overexpression of STAT3 is correlated with chemoresistance and radioresistance in NSCLC cells (65). Further studies may clarify the role of STAT3 in gefitinib resistance in NSCLC. In addition, shikonin suppresses EGFR phosphorylation and increases EGFR proteasomal degradation in NSCLC (92). Decreasing EGFR expression inhibits its downstream signaling. $\mathrm{Cbl}$ is a RING E3/protein-ubiquitin ligase recruited to EGFR that mediates EGFR degradation. However, the role of Cbl in shikonin-mediated EGFR degradation has not been studied.

Tanshinone IIA. Tanshinone IIA is a diterpenoid naphthoquinone derived from the roots of Salvia miltiorrhiza. A recent study shows that it synergistically enhances the cytotoxic activity of geftinib in gefitinib-resistant NSCLC cells (98). Both in vitro and in vivo studies suggest that tanshinone IIA potentiates the anti-proliferative and anti-metastatic effects of geftinib in TKI-resistant cancer cells, which is attributed to the increased level of cleaved caspase 3 and the inhibition of the VEGF receptor-2/Akt signaling pathway (98).

\section{Xanthones}

Gambogenic acid. Gambogenic acid is a polyprenylated xanthone isolated from the traditional Chinese medicinal herb gamboge. Gambogenic acid induces apoptosis in NSCLC cells by inhibiting the JAK/STAT3 signaling pathways (99); it also triggers autophagy by initiating fusion between autophagosomes and lysosomes, and inhibiting acidification in lysosomes, leading to cancer cell death (100). Furthermore, in a previous study, gambogenic acid abrogated the resistance to erlotinib in NSCLC, as demonstrated in a xenograft mouse model and patient-derived xenograft model by inhibiting c-Met activity and decreasing EGFR phosphorylation (99).

Gambogenic acid also overcomes resistance to cisplatin in NSCLC. In cisplatin-resistant NSCLC cells, gambogenic acid arrests the cell cycle at the $\mathrm{G}_{1}$ phase by decreasing the expression of cyclin D1, cyclin dependent kinase (CDK) 4 and CDK6, and upregulating p53 and p21. Moreover, gambogenic acid induces apoptosis by activating caspase-3 and -7 (101).

\section{Nucleosides}

Cordycepin. Cordyceps species such as C. Sinensis are traditional medicinal fungi. The main constituent of the extract derived from this fungus is cordycepin $(102,103)$. Cordycepin, or 3'-deoxyadenosine, is a derivative of the nucleoside adenosine, differing from the nucleoside only by the absence of the hydroxy group in the 3 ' position of its ribose moiety. Cordycepin was first found in fermented broth of the medicinal mushroom Cordyceps militaris (102). Cordycepin induces cell cycle arrest at the $\mathrm{G}_{0} / \mathrm{G}_{1}$ phase and apoptosis in lung cancer cells by inhibiting the phosphorylation of EGFR, Akt and ERK1/2 (104), and by interacting with and activating AMP-activated protein kinase $(105,106)$. Cordycepin is also capable of inhibiting NSCLC cell cycle progression. NSCLC cells containing EGFR mutations are more sensitive to cordycepin treatment than those without EGFR mutations. More importantly, the therapeutic potency of cordycepin is comparable to afatinib and gefitinib, suggesting cordycepin itself, or in combination with gefitinib, can be a novel therapeutic for treating drug-resistant NSCLC (107).

\section{Perspective}

Preclinical studies show that many bioactive compounds in the family of saponins, terpenoids, polyphenols and alkaloids overcome EGFR-TKI resistance in NSCLC via different mechanisms of action, such as the induction of apoptosis, autophagy and necroptosis, increased EGFR degradation, regulation of protein kinases and signaling activities, and the inhibition of transcription factors such as STAT3 and $\mathrm{NF}-\mathrm{kB}$.

Recently, studies have revealed other potential mechanisms that may underlie the development of EGFR-TKI resistance in NSCLC. Targeting these novel mechanisms and therapeutic targets may be a novel pragmatic approach to discover new drugs that can overcome EGFR-TKI resistance. For example, ubiquitin-specific peptidase 8 (USP8) may be a novel therapeutic target in gefitinib-resistant NSCLC cells. Knockdown of USP8 selectively kills gefitinib-resistant NSCLCs. It has been reported that synthetic USP8 inhibitor markedly decreases the viability of gefitinib-resistant and gefitinib-sensitive NSCLC cells by decreasing receptor tyrosine kinase expression while having no effect on normal cells (108). Ghrelin receptor could be another therapeutic target. Ghrelin receptor inhibitor reverses gefitinib resistance in NSCLC. A previous study showed that treating gefitinib-resistant NSCLC with antagonist for ghrelin-R (D-lys-3-GHRP-6) significantly decreased the phosphorylation of AKT and ERK1/2, inhibited cell proliferation and induced apoptosis (109). Inhibiting autophagy also enhances gefitinib cytotoxicity in gefitinib-resistant NSCLC cells. Computational protein-ligand docking and virtual drug screening with the AutoDock suite or the Local Move Monte Carlo-based approach may help to provide a direct and rational drug discovery with known therapeutic targets. These approaches allow us to model the interaction between 
a small molecule and a protein at the atomic level, and characterize the behavior of the small molecule in the binding site of the target proteins $(110,111)$. Subsequent functional studies can validate the function of the binding between the target protein and the small molecules or the bioactive compounds in NSCLC cells. Furthermore, post-translational modification of EGFR may also help to overcome resistance to EGFR-TKI. Upon EGF-binding, EGFR undergoes conformational changes and dimerization, resulting in kinase activation, autophosphorylation and activation of downstream signaling molecules. Sialylation, the covalent addition of sialic acid to the terminal end of glycoproteins, suppresses EGFR dimerization and EGFR phosphorylation. In EGFR-TKI-resistant NSCLC cells harboring EGFR L858R/T790M mutations, sialylation partially suppresses the phosphorylation at Y1068, Y1086 and Y1173 of EGFR and enhances EGFR sensitivity to EGRF-TKI (112). Therefore, screening bioactive compounds that can enhance sialylation in NSCLC may also overcome the resistance to EGFR-TKI treatment.

Targeting NF- $\mathrm{B}$ and cyclooxygenase-2 (COX-2) may also be an alternative approach to overcome EGFR resistance. EGFR mutations have been reported to be associated with increased COX-2 expression in NSCLC (113). Notably, there is increased downregulation of COX-2 and EGFR phosphorylation when NSCLC cells are treated with celecoxib and gefitinib compared with that when treated with either agent alone (113).

The upregulation of COX-2 in NSCLC may also be attributed to enhanced $N F-\kappa B$ activity. $N F-\kappa B$ activity is elevated in lung cancer and is considered as a therapeutic target (114). A number of studies have reported that COX-2 is a downstream target of NF- $\mathrm{B}$ in various cancer types, including lung cancer (115-117). Therefore, suppressing NF- $\kappa$ B activity will also decrease COX2 expression. Numerous natural compounds inhibit NF- $\kappa \mathrm{B}$ activity, including the triptolide isolated from Tripterygium wilfordii (118), Coix lacryma-jobi (119) and usolic acid (120). Additionally, epigallocatechin-3-gallate, genistein, luteolin, silibinin, deguelin, gallic acid, parthenolide, flavopiridol, anthocyanin, quinoclamine, dehydroxymethylepoxyquinomicin, guggulsterone, betulinic acid, emodin, gingerol, flavopiridol, zerumbone, indole-3-carbinol, elagic acid, anethole, green tea catechins, S-allyl cysteine, lycopene, genistein, quercetagenin, sasanquol, leuteolin, apigenin, wogonin and diosgenin also have NF- $\mathrm{B}$ suppressive functions (121-123). Among these compounds, some of the more well-known compounds such as berberine can suppress the nuclear translocation of $\mathrm{p} 50 / \mathrm{p} 65 \mathrm{NF}-\kappa \mathrm{B}$ proteins and the binding of $\mathrm{NF}-\kappa \mathrm{B}$ to the COX-2 promoter, thus decreasing COX-2 expression (124). Curcumin and other curcuminoids from the ginger family can regulate the activity of $\mathrm{NF}-\kappa \mathrm{B}$ and therefore the expression of the NF-kB regulated gene products, including COX-2, in NSCLC (125). Resveratrol has been reported to suppress TNF- $\alpha$-induced phosphorylation and nuclear trans-

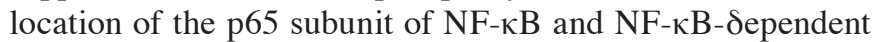
reporter gene transcription in lung cancer (126).

\section{Conclusion}

EGFR-TKIresistance underlines the failure of frontline NSCLC treatments. Natural herbal resources provide a vast pool for screening compound candidates that can specifically overcome EGFR-TKI resistance in NSCLC. Subsequent preclinical and clinical studies will help to develop these natural compounds as adjunct therapies for treating EGFR-TKI-resistant NSCLC and will benefit the affected patients.

\section{Acknowledgements}

Not applicable.

\section{Funding}

This study was partially supported by Research Grant Council of HKSAR=Hong Kong Special Administrative Region (grant no. HKBU-22103017-ECS), the Innovation \& Technology Commission (grant no. \#PRP/015/19FX), the National Natural ScienceFoundation of China(grantnos.\#SCM-2016-NSFC-003, 82074019 and 81703705), the Natural Science Foundation of Guangdong Province (grant no. \#2018A0303130122), Characteristic innovation projects of Universities in Guangdong Province (grant no. 2020KTSCX030), and the Opening Project of Zhejiang Provincial Preponderant and Characteristic Subject of Key University (Traditional Chinese Pharmacology), Zhejiang Chinese Medical University (grant no. ZYAOX2018010).

\section{Availability of data and materials}

Not applicable.

\section{Authors' contributions}

HYJL, YL, TS and HYK were responsible for conceptualization. Data curation and formal analysis were completed by HYJL and MM. HYJL, TS and HYK wrote the original manuscript. TS and HYK reviewed the manuscript. All authors have read and approved the manuscript. Data authentication is not applicable.

\section{Ethics approval and consent to participate}

Not applicable.

\section{Patient consent for publication}

Not applicable.

\section{Competing interests}

The authors declare that they have no competing interests.

\section{References}

1. Cao M and Chen W: Epidemiology of lung cancer in China. Thoracic Cancer 10: 3-7, 2019.

2. Zappa C and Mousa SA: Non-small cell lung cancer: Current treatment and future advances. Transl Lung Cancer Res 5: 288-3000, 2016.

3. Santoni-Rugiu E, Melchior LC, Urbanska EM, Jakobsen JN, Stricker K, Grauslund M and Sørensen JB: Intrinsic resistance to EGFR-tyrosine kinase inhibitors in EGFR-mutant non-small cell lung cancer: Differences and similarities with acquired resistance. Cancers (Basel) 11: 923, 2019. 
4. Wang Z: ErbB receptors and cancer. Methods Mol Biol 1652: 3-35, 2017.

5. Mok TS, Wu YL, Thongprasert S, Yang CH, Chu DT, Saijo N, Sunpaweravong P, Han B, Margono B, Ichinose Y, et al: Gefitinib or carboplatin-paclitaxel in pulmonary adenocarcinoma. N Engl J Med 361: 947-957, 2009.

6. Mitsudomi T, Morita S, Yatabe Y, Negoro S, Okamoto I, Tsurutani J, Seto T, Satouchi M, Tada H, Hirashima T, et al: Gefitinib versus cisplatin plus docetaxel in patients with non-small-cell lung cancer harbouring mutations of the epidermal growth factor receptor (WJTOG3405): An open label, randomised phase 3 trial. Lancet Oncol 11: 121-128, 2010.

7. Wang J, Wu Y, Dong M, He X, Wang Z, Li J and Wang Y: Observation of hepatotoxicity during long-term gefitinib administration in patients with non-small-cell lung cancer. Anti-cancer Drugs 27: 245-250, 2016.

8. Califano R, Tariq N, Compton S, Fitzgerald DA, Harwood CA, Lal R, Lester J, McPhelim J, Mulatero C, Subramanian S, et al: Expert consensus on the management of adverse events from EGFR tyrosine kinase inhibitors in the UK. Drugs 75: 1335-1348, 2015.

9. Ramalingam SS, Vansteenkiste J, Planchard D, Cho BC, Gray JE, Ohe Y, Zhou C, Reungwetwattana T, Cheng Y, Chewaskulyong B, et al: Overall survival with osimertinib in untreated, EGFR-mutated advanced NSCLC. N Engl J Med 382: 41-50, 2020.

10. Jackman D, Pao W, Riely GJ, Engelman JA, Kris MG, Jänne PA, Lynch T, Johnson BE and Miller VA: Clinical definition of acquired resistance to epidermal grow th factor receptor tyrosine kinase inhibitors in non-small-cell lung cancer. J Clin Oncol 28: 357-360, 2010.

11. Camidge DR, Pao W and Sequist LV: Acquired resistance to TKIs in solid tumours: Learning from lung cancer. Nat Rev Clin Oncol 11: 473-481, 2014.

12. Cancer Genome Atlas Research Network: Comprehensive molecular profiling of lung adenocarcinoma. Nature 511: 543-550, 2014

13. Cancer Genome Atlas Research Network: Comprehensive genomic characterization of squamous cell lung cancers. Nature 489: 519, 2012,2012.

14. Imielinski M, Berger AH, Hammerman PS, Hernandez B, Pugh TJ, Hodis E, Cho J, Suh J, Capelletti M, Sivachenko A, et al: Mapping the hallmarks of lung adenocarcinoma with massively parallel sequencing. Cell 150: 1107-1120, 2012.

15. Hansen RN, Zhang Y, Seal B, Ryan K, Yong C, Darilay A and Ramsey SD: Long-term survival trends in patients with unresectable stage III non-small cell lung cancer receiving chemotherapy and radiation therapy: A SEER cancer registry analysis. BMC Cancer 20: 276, 2020

16. Sui X, Zhang M, Han X, Zhang R, Chen L, Liu Y, Xiang Y and Xie T: Combination of traditional Chinese medicine and epidermal growth factor receptor tyrosine kinase inhibitors in the treatment of non-small cell lung cancer: A systematic review and meta-analysis. Medicine (Baltimore) 99: e20683, 2020.

17. Jiao L, Xu J, Sun J, Chen Z, Gong Y, Bi L, Lu Y, Yao J, Zhu W, Hou A, et al: Chinese herbal medicine combined with EGFR-TKI in EGFR mutation-positive advanced pulmonary adenocarcinoma (CATLA): A multicenter, randomized, double-blind, placebo-controlled trial. Front Pharmacol 10: 732, 2019.

18. Liu ZL, Zhu WR, Zhou WC, Ying HF, Zheng L, Guo YB, Chen JX and Shen XH: Traditional Chinese medicinal herbs combined with epidermal growth factor receptor tyrosine kinase inhibitor for advanced non-small cell lung cancer: A systematic review and meta-analysis. J Integr Med 12: 346-358, 2014.

19. Li CL, Hsia TC, Li CH, Chen KJ, Yang YH and Yang ST: Adjunctive traditional Chinese medicine improves survival in patients with advanced lung adenocarcinoma treated with first-line epidermal growth factor receptor (EGFR) tyrosine kinase inhibitors (TKIs): A nationwide, population-based cohort study. Integr Cancer Ther 18: 1534735419827079, 2019.

20. Yang XB, Wu WY, Long SQ, Deng H and Pan ZQ: Effect of gefitinib plus Chinese herbal medicine $(\mathrm{CHM})$ in patients with advanced non-small-cell lung cancer: A retrospective case-control study. Complement Ther Med 22: 1010-1018, 2014.

21. Tang M, Wang S, Zhao B, Wang W, Zhu Y, Hu L, Zhang X and Xiong S: Traditional Chinese medicine prolongs progression-free survival and enhances therapeutic effects in epidermal growth factor receptor tyrosine kinase inhibitor (EGFR-TKI) treated non-small-cell lung cancer (NSCLC) patients harboring EGFR mutations. Med Sci Monit 25: 8430-8437, 2019.

22. Wang CY, Huang HS, Su YC, Tu CY, Hsia TC and Huang ST: Conventional treatment integrated with Chinese herbal medicine improves the survival rate of patients with advanced non-small cell lung cancer. Complement Ther Med 40: 29-36, 2018.
23. Bing Z, Cheng Z, Shi D, Liu X, Tian J, Yao X, Zhang J, Wang Y and Yang K: Investigate the mechanisms of Chinese medicine Fuzhengkangai towards EGFR mutation-positive lung adenocarcinomas by network pharmacology. BMC Complement Altern Med 18: 293, 2018.

24. Xu W, Jiang X, Xu Z, Ye T and Shi Q: The efficacy of Brucea javanica oil emulsion injection as adjunctive therapy for advanced non-small-cell lung cancer: A meta-analysis. Evid Based Complement Alternat Med 2016: 5928562, 2016.

25. Kim SH, Liu CY, Fan PW, Hsieh CH, Lin HY, Lee MC and Fang K: The aqueous extract of Brucea javanica suppresses cell growth and alleviates tumorigenesis of human lung cancer cells by targeting mutated epidermal growth factor receptor. Drug Des Devel Ther 10: 3599-3609, 2016.

26. Ji ZQ, Huang XE, Wu XY, Liu J, Wang L and Tang JH: Safety of Brucea javanica and cantharidin combined with chemotherapy for treatment of NSCLC patients. Asian Pac J Cancer Prev 15: 8603-8605, 2014

27. Han SY, Zhao MB, Zhuang GB and Li PP: Marsdenia tenacissima extract restored gefitinib sensitivity in resistant non-small cell lung cancer cells. Lung Cancer 75: 30-37, 2012.

28. Han SY, Zhao W, Sun H, Zhou N, Zhou F, An G and Li PP: Marsdenia tenacissima extract enhances gefitinib efficacy in non-small cell lung cancer xenografts. Phytomedicine 22: 560-567, 2015.

29. Choi YJ, Kim JH, Rho JK, Kim JS, Choi CM, Kim WS, Son J and Lee JC: AXL and MET receptor tyrosine kinases are essential for lung cancer metastasis. Oncol Rep 37: 2201-2208, 2017.

30. Han SY, Sun H, Xue D, Zhao W, Jiao YN and Li PP: Overcomes AXL and Met mediated erlotinib/gefitinib cross resistance in non-small cell lung cancer cells by Marsdenia tenacissima extract. AACR: Jul 17, 2017 (Epub ahead of print). doi: 10.1158/1538-7445.AM2017-1199.

31. Deng J, Shen F and Chen D: Quantitation of seven polyoxypregnane glycosides in Marsdenia tenacissima using reversed-phase high-performance liquid chromatography-evaporative light-scattering detection. J Chromatogr A 1116: 83-88, 2006.

32. Wang Y, Chen B, Wang Z, Zhang W, Hao K, Chen Y, Li K, Wang T, Xie Y, Huang Z and Tong X: Marsdenia tenacissimae extraction (MTE) inhibits the proliferation and induces the apoptosis of human acute $\mathrm{T}$ cell leukemia cells through inactivating $\mathrm{PI} 3 \mathrm{~K} / \mathrm{AKT} / \mathrm{mTOR}$ signaling pathway via PTEN enhancement. Oncotarget 7: 82851-82863, 2016.

33. Song J, Zhong R, Huang H, Zhang Z, Ding D, Yan H, Sun E and Jia X: Combined treatment with Epimedium koreanum Nakai extract and gefitinib overcomes drug resistance caused by T790M mutation in non-small cell lung cancer cells. Nutr Cancer 66: 682-689, 2014.

34. Cheng Y, Wang X, Zhang DW, Wang NL and Yao XS: Nonflavanoid compounds from Epimedium koreanum. Chin Tradit Herb Drugs 38: 1135-1138, 2007.

35. Yu X, Tong Y, Han XQ, Kwok HF, Yue GG, Lau CB and Ge W: Anti-angiogenic activity of herba epimedii on zebrafish embryos in vivo and HUVECs in vitro. Phytother Res 27: 1368-1375, 2013.

36. Salehi B, Mishra AP, Nigam M, Sener B, Kilic M, Sharifi-Rad M, Fokou PVT, Martins N and Sharifi-Rad J: Resveratrol: A Double-Edged sword in health benefits. Biomedicines 6: 91, 2018.

37. Zhu Y, He W, Gao X, Li B, Mei C, Xu R and Chen H: Resveratrol overcomes gefitinib resistance by increasing the intracellular gefitinib concentration and triggering apoptosis, autophagy and senescence in PC9/G NSCLC cells. Sci Rep 5: 17730, 2015.

38. Nam B, Rho JK, Shin D and Son J: Gallic acid induces apoptosis in EGFR-mutant non-small cell lung cancers by accelerating EGFR turnover. Bioorg Med Chem Lett 26: 4571-4575, 2016.

39. Phan AN, Hua TN, Kim MK, Vo VT, Choi JW, Kim HW, Rho JK, Kim KW and Jeong Y: Gallic acid inhibition of Src-Stat3 signaling overcomes acquired resistance to EGF receptor tyrosine kinase inhibitors in advanced non-small cell lung cancer. Oncotarget 7: 54702-54713, 2016.

40. Jin H, Qiao F, Wang Y, Xu Y and Shang Y: Curcumin inhibits cell proliferation and induces apoptosis of human non-small cell lung cancer cells through the upregulation of miR-192-5p and suppression of PI3K/Akt signaling pathway. Oncol Rep 34: 2782-2789, 2015.

41. Lu Y, Wei C and Xi Z: Curcumin suppresses proliferation and invasion in non-small cell lung cancer by modulation of MTA1-mediated Wnt/ $\beta$-catenin pathway. In Vitro Cell Dev Biol Anim 50: 840-850, 2014.

42. Li Y, Chao Y, Fang Y, Wang J, Wang M, Zhang H, Ying M, Zhu X and Wang H: MTA1 promotes the invasion and migration of non-small cell lung cancer cells by downregulating miR-125b. J Exp Clin Cancer Res 32: 33, 2013. 
43. Xiao K, Jiang J, Guan C, Dong C, Wang G, Bai L, Sun J, Hu C and Bai C: Curcumin induces autophagy via activating the AMPK signaling pathway in lung adenocarcinoma cells. J Pharmacol Sci 123: 102-109, 2013

44. Lee J, Lee Y, Chang G, Yu SL, Hsieh WY, Chen JJ, Chen HW and Yang PC: Curcumin induces EGFR degradation in lung adenocarcinoma and modulates p38 activation in intestine: The versatile adjuvant for gefitinib therapy. PLoS One 6: e23756, 2011.

45. Chen P, Huang H, Wang Y, Jin J, Long WG, Chen K, Zhao XH, Chen CG and Li J: Curcumin overcome primary gefitinib resistance in non-small-cell lung cancer cells through inducing autophagy-related cell death. J Exp Clin Cancer Res 38: 254, 2019.

46. Yamauchi Y,Izumi Y, Yamamoto Jand Nomori H: Coadministration of erlotinib and curcumin augmentatively reduces cell viability in lung cancer cells. Phytother Res 28: 728-735, 2014.

47. Tran K, Merika M and Thanos D: Distinct functional properties of IkappaB alpha and IkappaB beta. Mol Cell Biol 17: 5386-5399, 1997.

48. Li S, Liu Z, Zhu F, Fan X, Wu X, Zhao H and Jiang L: Curcumin lowers erlotinib resistance in non-small cell lung carcinoma cells with mutated EGF receptor. Oncol Res 21: 137-144, 2014.

49. Ye MX, Zhao YL, Li Y, Miao Q, Li ZK, Ren XL, Song LQ, Yin $\mathrm{H}$ and Zhang J: Curcumin reverses cis-platin resistance and promotes human lung adenocarcinoma A549/DDP cell apoptosis through HIF-1 $\alpha$ and caspase-3 mechanisms. Phytomedicine 19: 779-787, 2012

50. Chanvorachote P, Pongrakhananon V, Wannachaiyasit S, Luanpitpong S, Rojanasakul Y and Nimmannit U: Curcumin sensitizes lung cancer cells to cisplatin-induced apoptosis through superoxide anion-mediated Bcl-2 degradation. Cancer Invest 27: 624-635, 2009.

51. Zheng M, Xin Y, Li Y, Xu F, Xi X, Guo H, Cui X, Cao H, Zhang $\mathrm{X}$ and Han C: Ginsenosides: A potential neuroprotective agent. Biomed Res Int 2018: 8174345, 2018.

52. Liu T, Zuo L, Guo D, Chai X, Xu J, Cui Z, Wang Z and Hou C: Ginsenoside $\mathrm{Rg} 3$ regulates DNA damage in non-small cell lung cancer cells by activating VRK1/P53BP1 pathway. Biomed Pharmacother 120: 109483, 2019.

53. Dai Y, Wang W, Sun Q and Tuohayi J: Ginsenoside Rg3 promotes the antitumor activity of gefitinib in lung cancer cell lines. Exp Ther Med 17: 953-959, 2018.

54. Nieto MA: The snail superfamily of zinc-finger transcription factors. Nat Rev Mol Cell Biol 3: 155-166, 2002.

55. Zhang F, Wang J, Wang X, Wei N, Liu H and Zhang X: CD146mediated acquisition of stemness phenotype enhances tumour invasion and metastasis after EGFR-TKI resistance in lung cancer. Clin Respir J 13: 23-33, 2019.

56. Tan Q, Lin S, Zeng Y, Yao M, Liu K, Yuan H, Liu C and Jiang G: Ginsenoside Rg3 attenuates the osimertinib resistance by reducing the stemness of non-small cell lung cancer cells Environ Toxicol 35: 643-651, 2020

57. Xu T, Jin Z, Yuan Y, Wei H, Xu X, He S, Chen S, Hou W, Guo Q and Hua B: Ginsenoside Rg3 serves as an adjuvant chemotherapeutic agent and VEGF inhibitor in the treatment of non-small cell lung cancer: A meta-analysis and systematic review. Evid Based Complement Alternat Med 2016: 7826753, 2016.

58. Chian S, Zhao Y, Xu M, Yu X, Ke X, Gao R and Yin L: Ginsenoside $\mathrm{Rd}$ reverses cisplatin resistance in non-small-cell lung cancer A549 cells by downregulating the nuclear factor erythroid 2-related factor 2 pathway. Anticancer Drugs 30: 838-845, 2019.

59. Jiang Z, Yang Y, Yang Y, Zhang Y, Yue Z, Pan Z and Ren X: Ginsenoside Rg3 attenuates cisplatin resistance in lung cancer by downregulating PD-L1 and resuming immune. Biomed Pharmacother 96: 378-383, 2017.

60. Dai P, Liu D, Zhang L, Ye J, Wang Q, Zhang HW, Lin XH and Lai GX: Astragaloside IV sensitizes non-small cell lung cancer cells to gefitinib potentially via regulation of SIRT6. Tumor Biol: Apr 26, 2017 (Epub ahead of print). doi: 10.1177/1010428317697555.

61. Yang Q, Chen W, Xu Y, Lv X, Zhang M and Jiang H: Polyphyllin I modulates MALAT1/STAT3 signaling to induce apoptosis in gefitinib-resistant non-small cell lung cancer. Toxicol App Pharmacol 356: 1-7, 2018

62. Tang Y, Xiao G, Chen Y and Deng Y: LncRNA MALAT1 promotes migration and invasion of non-small-cell lung cancer by targeting miR-206 and activating Akt/mTOR signaling. Anticancer Drugs 29: 725-735, 2018

63. Li S, Mei Z, Hu H and Zhang X: The lncRNA MALAT1 contributes to non-small cell lung cancer development via modulating miR-124/STAT3 axis. J Cell Physiol 233: 6679-6688, 2018.
64. Amodio N, Raimondi L, Juli G, Stamato MA, Caracciolo D, Tagliaferri P and Tassone P: MALAT1: A druggable long non-coding RNA for targeted anti-cancer approaches. J Hematol Oncol 11: 63, 2018

65. Harada D, Takigawa N and Kiura K: The role of STAT3 in non-small cell lung cancer. Cancers (Basel) 6: 708-722, 2014

66. Xu YH and Lu S: A meta-analysis of STAT3 and phospho-STAT3 expression and survival of patients with non-small-cell lung cancer. Eur J Surg Oncol 40: 311-317, 2014.

67. Zulkifli AA, Tan FH, Putoczki TL, Stylli SS and Luwor RB: STAT3 signaling mediates tumour resistance to EGFR targeted therapeutics. Mol Cell Endocrinol 451: 15-23, 2017.

68. Zheng R, Jiang H, Li J, Liu X and Xu H: Polyphyllin II restores sensitization of the resistance of PC-9/ZD cells to gefitinib by a negative regulation of the $\mathrm{PI} 3 \mathrm{~K} / \mathrm{Akt} / \mathrm{mTOR}$ signaling pathway. Curr Cancer Drug Targets 17: 376-385, 2017.

69. Wang H, Fei Z and Jiang H: Polyphyllin VII increases sensitivity to gefitinib by modulating the elevation of P21 in acquired gefitinib resistant non-small cell lung cancer. J Pharmacol Sci 134: 190-196, 2017.

70. Lou W, Chen Y, Zhu K, Deng H, Wu T and Wang J: Polyphyllin I overcomes EMT-associated resistance to erlotinib in lung cancer cells via IL-6/STAT3 pathway inhibition. Biol Pharm Bull 40: $1306-1313,2017$

71. Feng F, Cheng P, Sun C, Wang $\mathrm{H}$ and Wang W: Inhibitory effects of polyphyllins I and VII on human cisplatin-resistant NSCLC via p53 upregulation and CIP2A/AKT/mTOR signaling axis inhibition. Chin J Nat Med 17: 768-777, 2019.

72. Feng F, Cheng P, Wang C, Wang Y and Wang W: Polyphyllin I and VII potentiate the chemosensitivity of A549/DDP cells to cisplatin by enhancing apoptosis, reversing EMT and suppressing the CIP2A/AKT/mTOR signaling axis. Oncol Lett 18: 5428-5436, 2019.

73. Wang YC, Wu DW, Wu TC, Wang L, Chen CY and Lee $\mathrm{H}$ Dioscin overcome TKI resistance in EGFR-mutated lung adenocarcinoma cells via down-regulation of tyrosine phosphatase SHP2 expression. Int J Biol Sci 14: 47-56, 2018.

74. Mainardi S, Mulerosanchez A, Prahallad A, Germano G, Bosma A, Krimpenfort P, Lieftink C, Steinberg JD, de Wit N, Gonçalves-Ribeiro S, et al: SHP2 is required for growth of KRAS-mutant non-small-cell lung cancer in vivo. Nat Med 24: 961-967, 2018.

75. Zhang S, Xu Y, Jin E, Zhu LC, Xia B, Chen XF, Li FZ and Ma SL: Capilliposide from Lysimachia capillipes inhibits AKT activation and restores gefitinib sensitivity in human non-small cell lung cancer cells with acquired gefitinib resistance. Acta Pharmacol Sin 38: 100-109, 2017

76. Kausar H, Munagala R, Bansal SS, Aqil F, Vadhanam MV and Gupta RC: Cucurbitacin B potently suppresses non-small-cell lung cancer growth: Identification of intracellular thiols as critical targets. Cancer Letters 332: 35-45, 2013.

77. Li W, Liu Y, Cai S, Yang C, Lin Z, Zhou L, Liu L, Cheng X and Zeng W: Not all mutations of KRAS predict poor prognosis in patients with colorectal cancer. Int J Clin Exp Pathol 12: 957-967, 2019.

78. Wei L, Qu W, Sun J, Wang X, Lv L, Xie L and Song X: Knockdown of cancerous inhibitor of protein phosphatase $2 \mathrm{~A}$ may sensitize NSCLC cells to cisplatin. Cancer Gene Therapy 21: 194-199, 2014.

79. Sablina AA, Chen W, Arroyo JD, Corral L, Hector M, Bulmer SE, DeCaprio JA and Hahn WC: The tumor suppressor PP2A Abeta regulates the RalA GTPase. Cell 129: 969-982, 2007.

80. Liu P, Xiang Y, Liu X, Zhang T, Yang R, Chen S, Xu L, Yu Q, Zhao $\mathrm{H}$, Zhang L, et al: Cucurbitacin $\mathrm{B}$ induces the lysosomal degradation of EGFR and suppresses the CIP2A/PP2A/Akt signaling axis in gefitinib-resistant non-small cell lung cancer. Molecules 24: 647, 2019.

81. Hong SH, Ku JM, Lim YS, Lee SY, Kim JH, Cheon C and Ko SG: Cucurbitacin D overcomes gefitinib resistance by blocking EGF binding to EGFR and inducing cell death in NSCLCs. Front Oncol 10: 62, 2020.

82. Zhou G, Chen S, Wang Z and Chen Z: Back to the future of oridonin Again, compound from medicinal herb shows potent antileukemia efficacies in vitro and in vivo. Cell Res 17: 274-276, 2007.

83. Xiao X, He Z, Cao W, Cai F, Zhang L, Huang Q, Fan C, Duan C, Wang X, Wang J and Liu Y: Oridonin inhibits gefitinib-resistant lung cancer cells by suppressing EGFR/ERK/MMP-12 and CIP2A/Akt signaling pathways. Int J Oncol 48: 2608-2618, 2016.

84. Tong Y, Liu Y, Zheng H, Zheng L, Liu W, Wu J, Ou R, Zhang G, Li F, Hu M, et al: Artemisinin and its derivatives can significantly inhibit lung tumorigenesis and tumor metastasis through Wnt $/ \beta$-catenin signaling. Oncotarget 7 : $31413-31428,2016$. 
85. Zhang S, Wang Y, Dai SD and Wang EH: Down-regulation of NKD1 increases the invasive potential of non-small-cell lung cancer and correlates with a poor prognosis. BMC Cancer 11: 186, 2011.

86. Jho EH, Zhang T, Domon C, Joo CK, Freund JN and Costantini F: Wnt/beta-Catenin/Tcf signaling induces the transcription of Axin2, a negative regulator of the signaling pathway. Mol Cell Biol 22: 1172-1183, 2002.

87. Shen R, Li J, Ye D, Wang Q and Fei J: Combination of onconase and dihydroartemisinin synergistically suppresses growth and angiogenesis of non-small-cell lung carcinoma and malignant mesothelioma. Acta Biochim Biophys Sin (Shanghai) 48: 894-901, 2016.

88. Hong J, Jiang AY, Han W, Yong C, Yan W and Jiang XF: Dihydroartemisinin and gefitinib synergistically inhibit NSCLC cell growth and promote apoptosis via the Akt/mTOR/STAT3 pathway. Mol Med Rep 16: 3475-3481, 2017.

89. Chen SF, Zhang ZY and Zhang JL: Matrine increases the inhibitory effects of afatinib on H1975 cells via the IL-6/JAK1/STAT3 signaling pathway. Mol Med Rep 16: 2733-2739, 2017.

90. Wang HQ, Jin JJ and Wang J: Matrine induces mitochondrial apoptosis in cisplatin-resistant non-small cell lung cancer cells via suppression of $\beta$-catenin/survivin signaling. Oncol Rep 33 : 2561-2566, 2015.

91. Li W, Yu X, Tan S, Liu W, Zhou L and Liu H: Oxymatrine inhibits non-small cell lung cancer via suppression of EGFR signaling pathway. Cancer Med 7: 208-218, 2018

92.Li X, Fan XX, Jiang ZB, Loo WT, Yao XJ, Leung EL, Chow LW and Liu L: Shikonin inhibits gefitinib-resistant non-small cell lung cancer by inhibiting TrxR and activating the EGFR proteasomal degradation pathway. Pharmacol Res 115: 45-55, 2017.

93. Kim HJ, Hwang KE, Park DS, Oh SH, Jun HY, Yoon KH, Jeong ET, Kim HR and Kim YS: Shikonin-induced necroptosis is enhanced by the inhibition of autophagy in non-small cell lung cancer cells. J Transl Med 15: 123, 2017.

94. Li YL, Hu X, Li QY, Wang F and Zhang C: Shikonin sensitizes wild-type EGFR NSCLC cells to erlotinib and gefitinib therapy. Mol Med Rep 18: 3882-3890, 2018.

95. Tang JC, Ren YG, Zhao J, Long F, Chen JY and Jiang Z: Shikonin enhances sensitization of gefitinib against wild-type EGFR non-small cell lung cancer via inhibition PKM2/stat3/cyclinD1 signal pathway. Life Sci 204: 71-77, 2018.

96. Kobierzycki C, Pula B, Werynska B, Piotrowska A Muszczynska-Bernhard B, Dziegiel P and Rakus D: The lack of evidence for correlation of pyruvate kinase M2 expression with tumor grade in non-small cell lung cancer. Anticancer Res 34 3811-3817, 2014

97. Guo ZL, Li JZ, Ma YY, Qian D, Zhong JY, Jin MM, Huang P, Che LY, Pan B, Wang Y, et al: Shikonin sensitizes A549 cells to TRAIL-induced apoptosis through the JNK, STAT3 and AKT pathways. BMC Cell Biol 19: 29, 2018.

98. Wang R, Luo Z, Zhang $\mathrm{H}$ and Wang T: Tanshinone IIA reverses gefitinib-resistance in human non-small-cell lung cancer via regulation of VEGFR/Akt pathway. Onco Targets Ther 12 9355-9365, 2019

99. Xu L, Meng X, Xu N, Fu W, Tan H,Zhang L, Zhou Q, Qian J, Tu S, Li X, et al: Gambogenic acid inhibits fibroblast growth factor receptor signaling pathway in erlotinib-resistant non-small-cell lung cancer and suppresses patient-derived xenograft growth Cell Death Dis 9: 262, 2018

100. Mei W, Dong C, Hui C, Bin L, Fenggen Y, Jingjing S, Cheng P, Meiling S, Yawen H, Xiaoshan W, et al: Gambogenic acid kills lung cancer cells through aberrant autophagy. PLoS One 9: e83604, 2014

101. Shen D, Wang Y, Niu H and Liu C: Gambogenic acid exerts anticancer effects in cisplatin-resistant non-small cell lung cancer cells. Mol Med Rep 21: 1267-1275, 2020.

102. Ye J, Xue M, Qiu Z, Su Y, Yu P and Peng Q: Anti-tumor and anti-metastatic roles of cordycepin, one bioactive compound of Cordyceps militaris. Saudi J Biol Sci 25: 991-995, 2018.

103. Tuli HS, Sandhu SS and Sharma AK: Pharmacological and therapeutic potential of Cordyceps with special reference to Cordycepin. 3 Biotech 4: 1-12, 2014.

104. Wang Z, Wu X, Liang YN, Wang L, Song ZX, Liu JL and Tang ZS: Cordycepin induces apoptosis and inhibits proliferation of human lung cancer cell line H1975 via Inhibiting the Phosphorylation of EGFR. Molecules 21: 1267, 2016.

105. Hawley SA, Ross FA, Russell FM, Atrih A, Lamont DJ and Hardie DG: Mechanism of activation of AMPK by cordycepin. Cell Chem Biol 27: 214-222.e4, 2020.
106. Wang Z, Wang X, Qu K, Zhu P, Guo N, Zhang R, Abliz Z, $\mathrm{Yu} \mathrm{H}$ and $\mathrm{Zhu} \mathrm{H}$ : Binding of cordycepin monophosphate to AMP-activated protein kinase and its effect on AMP-activated protein kinase activation. Chem Biol Drug Des 76: 340-344, 2010.

107. Wei C, Yao X, Jiang Z, Wang Y, Zhang D, Chen X, Fan X, Xie C, Cheng J, Fu J and Leung EL: Cordycepin inhibits drug-resistance non-small cell lung cancer progression by activating AMPK signaling pathway. Pharmacol Res 144: 79-89, 2019.

108. Byun S, Lee SY, Lee J, Jeong CH, Farrand L, Lim S, Reddy K, Kim JY, Lee MH, Lee HJ, et al: USP8 is a novel target for overcoming gefitinib resistance in lung cancer. Clin Cancer Res 19: 3894-3904, 2013

109. Li X, Zhao X, Li C, Liu S, Yan F, Teng Y, Feng J and Miao D: Inhibitor of ghrelin receptor reverses gefitinib resistance in lung cancer. Human Cell 32: 360-366, 2019

110. Forli S, Huey R, Pique ME, Sanner MF, Goodsell DS and Olson AJ: Computational protein-ligand docking and virtual drug screening with the AutoDock suite. Nat Protocols 11 905-919, 2016

111. Meng XY, Zhang HX, Mezei M and Cui M: Molecular docking: A powerful approach for structure-based drug discovery. Curr Comput Aided Drug Des 7: 146-157, 2011

112. Yen HY, Liu YC, Chen NY, Tsai CF, Wang YT, Chen YJ, Hsu TL, Yang PC and Wong CH: Effect of sialylation on EGFR phosphorylation and resistance to tyrosine kinase inhibition. Proc Natl Acad Sci USA 112: 6955-6960, 2015.

113. Li N, Li HH, Su F, Li J, Ma XP and Gong P: Relationship between epidermal growth factor receptor (EGFR) mutation and serum cyclooxygenase-2 Level, and the synergistic effect of celecoxib and gefitinib on EGFR expression in non-small cell lung cancer cells. Int J Clin Exp Pathol 8: 9010-9020, 2015.

114. Chen W, Li Z, Bai L and Lin Y: NF-kappaB in lung cancer, a carcinogenesis mediator and a prevention and therapy target. Front Biosci (Landmark Ed) 16: 1172-1185, 2011.

115. Dai M, Hu S, Liu CF, Jiang L, Yu W, Li ZL, Guo W, Tang R, Dong CY, Wu TH and Deng WG: BPTF cooperates with p50 $\mathrm{NF}-\mathrm{KB}$ to promote $\mathrm{COX}-2$ expression and tumor cell growth in lung cancer. Am J Transl Res 11: 7398-7409, 2019.

116. Konson A, Mahajna JA, Danon A, Rimon G and Agbaria R The involvement of nuclear factor-kappa B in cyclooxygenase-2 overexpression in murine colon cancer cells transduced with herpes simplex virus thymidine kinase gene. Cancer Gene Ther 13: 1093-1104, 2006.

117. Shi G, Li D, Fu J, Sun Y, Li Y, Qu R, Jin X and Li D: Upregulation of cyclooxygenase- 2 is associated with activation of the alternative nuclear factor kappa B signaling pathway in colonic adenocarcinoma. Am J Transl Res 7: 1612-1620, 2015.

118. Lee KY, Park JS, Jee YK and Rosen GD: Triptolide sensitizes lung cancer cells to TNF-related apoptosis-inducing ligand (TRAIL)-induced apoptosis by inhibition of NF-kappaB activation. Exp Mol Med 34: 462-468, 2002.

119. Woo JH,LiDP, Wilsbach K, Orita H, Coulter J, Tully E, Kwon TK $\mathrm{Xu} \mathrm{S}$ and Gabrielson E: Coix seed extract, a commonly used treatment for cancer in china, inhibits NFkappaB and protein kinase C signaling. Cancer Biol Ther 6: 2005-2011, 2007.

120. Hsu YL, Kuo PL and Lin CC: Proliferative inhibition, cell-cycle dysregulation, and induction of apoptosis by ursolic acid in human non-small cell lung cancer A549 cells. Life Sci 75: 2303-2316, 2004.

121. Lin Y, Shi RX, Wang X and Shen HM: Luteolin, a flavonoid with potential for cancer prevention and therapy. Curr Cancer Drug Targets 8: 634-646, 2008

122. Birt DF, Hendrich S and Wang WQ: Dietary agents in cancer prevention: Flavonoids and isoflavonoids. Pharmacol Ther 90: 157-177, 2001.

123. Aggarwal BB and Shishodia S: Molecular targets of dietary agents for prevention and therapy of cancer. Biochem Pharmacol 71: 1397-1421, 2006.

124. Fu L, Chen W, Guo W, Wang J, Tian Y, Shi D, Zhang X, Qiu H, Xiao X, Kang T, et al: Berberine targets AP-2/hTERT, $\mathrm{NF}-\kappa \mathrm{B} / \mathrm{COX}-2, \mathrm{HIF}-1 \alpha / \mathrm{VEGF}$ and Cytochrome-c/Caspase signaling to suppress human cancer cell growth. PLoS One 8: e69240, 2013

125. Tsai JR, Liu PL, Chen YH, Chou SH, Cheng YJ, Hwang JJ and Chong IW: Curcumin inhibits non-small cell lung cancer cells metastasis through the Adiponectin/NF- $\mathrm{kb} / \mathrm{MMPs}$ signaling pathway. PLoS One 10: e0144462, 2015.

126. Wright C, Iyer AKV, Yakisich JS and Azad N: Anti-tumorigenic effects of resveratrol in lung cancer cells through modulation of c-FLIP. Curr Cancer Drug Targets 17: 669-680, 2017. 